UDK: 811.134.2'367

DOI: https://doi.org/10.18485/legado_hispanico.2020.ch19

\author{
Tibor Berta ${ }^{1}$ \\ Universidad de Szeged \\ Hungría
}

\title{
LA SELECCIÓN DE AUXILIARES DE VERBOS INTRANSITIVOS EN LA VERSIÓN SEVILLANA DE LA HISTORIA DEL NOBLE VESPASIANO
}

\begin{abstract}
Resumen
El objetivo de este estudio es examinar la alternancia de auxiliares distintos - correspondientes a los latinos HABERE y ESSE- en las construcciones formadas con el participio de verbos intransitivos, que, como en otras lenguas que la conservan hasta hoy, también existió en el español antiguo. Tanto las condiciones de variación de los verbos como la cronología de su desaparición han sido abundantemente examinadas, pero los datos y las conclusiones de la bibliografía especializada son, de vez en cuando, contradictorios. En este trabajo nos proponemos analizar los datos extraídos de la versión sevillana del texto narrativo de la Historia del noble Vespasiano (1499), con el objetivo de clasificar los verbos intransitivos del español antiguo según la selección de auxiliar, prestando atención especial a aquellos que muestran alternancia aceptando ambos auxiliares.
\end{abstract}

Palabras clave: selección de auxiliares, tiempos verbales compuestos, español antiguo.

$\overline{{ }^{1} \text { tberta@hist.u-szeged.hu }}$ 
El objetivo de este trabajo es examinar la configuración de los tiempos verbales compuestos en construcciones intransitivas desde el punto de vista de la selección de auxiliar en la edición sevillana de la Historia del noble Vespasiano (1499). Centraremos nuestra atención en el fenómeno de la llamada doble auxiliaridad, consistente en seleccionar auxiliares distintos - correspondientes a los latinos HABERE y ESSE- para las construcciones formadas con el participio que caracteriza diversas lenguas europeas, según se ve en los ejemplos citados en (1), procedentes del francés, del italiano y del neerlandés, tomados de Aranovich (2003: 2).
a. fr. Marie est arrivée.
b. fr. J'ai parlé.
c. it. Il bambino è morto.
d. it. Sandro ha sorriso.
e. nl. De kinderen zijn in Amsterdam gebleven.
f. nl. Janneke heeft geknipoogd.

Han surgido diversas hipótesis que han intentado describir los motivos de alternancia de auxiliares presentada, donde una parte de los verbos intransitivos selecciona el mismo auxiliar que se usa en estructuras transitivas, mientras que la otra acepta el auxiliar de los pasivos. La elección entre los dos auxiliares en opinión de Perlmutter $(1978,1989)$ y Burzio (1986) responde a una categorización sintáctica de los verbos según su carácter inergativo o inacusativo. Otros estudios destacan los aspectos semánticos que determinan la elección de auxiliar: esta según Vincent (1982) y Aranovich (2003) depende del carácter agentivo y no agentivo del sujeto, según Mithun (1991) es definida por la actividad o inactividad de este, mientras que según Sorace (2000) por parámetros aspectuales y temáticos. Esta última autora -teniendo en cuenta diversas lenguas- explica los casos de alternancia en el marco de su hipótesis que supone que existe una jerarquía en la selección de auxiliar: ciertos verbos -considerados por ella nucleares y situados en los dos extremos de una escala gradual-seleccionan categóricamente HABERE o ESSE teniendo en cuenta la agentividad, la dinamicidad y la telicidad, mientras que otros -llamados periféricos- aceptan en mayor o menor grado la variación entre ambos auxiliares.

Entre las lenguas románicas de la península Ibérica, en el catalán existió una alternancia similar, que se mantiene en ciertos dialectos de hoy, lo cual explica que haya sido examinado abundantemente -véanse Pérez Saldanya (1998: 213-214), Mateu Fontanals (2005: 220-225), 
Ramos (2005), Moll (2006: §481), Batlle et al. (2016: §12.7.3.)-. En el español antiguo también existió el mismo fenómeno - tal como muestran los ejemplos aducidos en (2-5), donde fablar, cavalgar y andar seleccionan aver, mientras que morir y nacer aparecen con ser-, de lo que encontramos referencias en obras de gramática histórica generales -como las de Yllera (1980: 230-245), Lapesa (2000: 784-787; Penny 2005: 193-194) - y análisis específicos -como los de Benzing (1931), Company (1983), Pountain (1985), Elvira (2001), García Martín (2001: 106-118), Aranovich (2003), Rodríguez Molina (2006), Romani (2006, 2008), Stolova (2006) y Rosemeyer (2012, 2014)-. Elvira (2001: 212 y ss.) y Rodríguez Molina (2006: 1066-1069), diferencian los casos donde la selección entre haber y ser distinguía categóricamente dos tipos de verbos - como en (2) y (3)-, de aquellos donde la variación del auxiliar afectaba el mismo verbo, respondiendo a una alternancia diatética inacusativa / transitiva -como en (4)-, o bien sin alternancia diatética -como en (5)-.

(2) a. et pues que ouieron fablado en una buena pieça del dia (CEM 238, citado por Romani 2008: 1078)

b. los ifantes de Carrión bien an cavalgado. (PMC 2246)

c. Porque por muchas tierras auia andado (APO 125d)

(3) a. Cuando las gentes lo vieron, cuidaron que era muerto, (ECL p. 131)

b. Poco tiempo ha que es nacida. (ARM 4)

(4) a. Passada es la noche, venida es la mañana (PMC 1540)

b. Andan los dias e las noches e passada han la sierra (PMC 1823)

c. Espidiénse al rey, con esto tornados son (PMC 1914)

d. A sos castiellos a los moros dentro los an tornados (PMC 801)

e. Entendió de su vida que era acabada (ALE p. 268)

f. Et desque aquel rey ovo acabada la mezquita (ECL p. 166)

(5) a. aún non era llegado el que en buen ora naçió (PMC 3013)

b. fata la çintura el espada llegado ha. (PMC 2424)

c. Entrados son a Molina, buena y rica casa (PMC 1530)

d. Tórnanse con las dueñas, a Valencia an entrado (PMC 2247)

El fenómeno en cuestión en el caso del español muestra ciertas particularidades tanto sincrónica como diacrónicamente.

Desde la perspectiva sincrónica cabe destacar que la selección de haber y ser, respectivamente, en el español antiguo fue menos categórica 
de lo que es en otras lenguas, puesto que los verbos que aceptaban ser también aceptaban con mayor o menor frecuencia haber (Lapesa 2000: 784, Elvira 2001, Octavio de Toledo 2002: 259, Penny 2006: 194, Suárez 2016: 158, entre otros). Parece que también existe relación entre la aceptación de haber y la posibilidad de usar el verbo intransitivo como transitivo (Yllera 1980: 231, citado por García Martín 2001: 109). Para algunos investigadores la alternancia de auxiliares documentada con el mismo verbo debió de tener un valor meramente estilístico (Keniston 1937: 450-451; Company 1983: 243-245; Lapesa 1988: 212), según otros, en cambio, se sometía a ciertas condiciones. Algunos atribuyen significados distintos a las dos construcciones considerando que con haber se presentaba la mera acción y con ser la acción con su resultado ${ }^{2}$ (Molho 1975: 178-181), Yllera (1980: 231-232; Andres-Suárez 1994: 72; Octavio de Toledo 2002: 260) o bien que la diferencia radicaba en si la construcción focalizaba la parte procesal o el resultado del evento (Arias Álvarez 2000: 210-211). Más recientemente se ha observado la preferencia por haber en casos de modalidad irreal y negación (Stolova $2006)^{3}$. La distribución funcional distinta de las construcciones con haber y con ser lleva a algunos autores a concluir que ser+participio en el español medieval no era tiempo verbal compuesto, sino construcción resultativa (Rodríguez Molina 2006), es decir, que se trata de dos tipos de estructura diferentes Rosemeyer $(2012,2014)^{4}$.

Desde el punto de vista diacrónico, destaca la gran variedad de información referente a la cronología de la desaparición del fenómeno en cuestión en la bibliografía especializada. Las referencias generales se limitan a afirmar que su decadencia se inició en el siglo XVI y se consumó en el XVII (Eberenz 1991: 99, 2009: 187; Lapesa 2000: 785; Penny 2006: 194), mientras que algunos estudios dedicados más específicamente a este tema atribuyen una importancia fundamental al siglo XV (Rosemeyer 2014: 138-141), cuando el uso de ser parece restringirse a ciertos verbos -nacer, pasar, llegar y venir- (Company 1983: 241-242). De hecho, los estudios específicos hacen constar que el proceso fue gradual y afectó

\footnotetext{
${ }^{2}$ Ya Par (1923: 320) -en lo referente al catalán antiguo- había afirmado que es «l’auxiliar ço qui nos indica com devem considerar l'acció». Octavio de Toledo cita la gramática española de Oudin publicada en 1597, que, a propósito de las formas yo he venido y yo soy venido afirma que «le premier signifie l'action \& mouvement de la venue; \& le second denote le repos apres le venue» (citado aquí según la edición de 1639: 145).

${ }^{3}$ Cennamo (2008) llega a la misma conclusión al analizar textos italianos antiguos.

${ }^{4}$ Textualmente, Rosemeyer (2014: 137) afirma que «in most cases, Old Spanish haber + PtcP and ser + PtcP are constructions of different types».
} 
diferentes tipos de verbos en épocas distintas: los verbos de estado, de existencia y de aparición serían los primeros, mientras que los de movimiento -ir, salir y venir- y de cambio de estado - morir, nacer y pasar- los últimos en perder la posibilidad de seleccionar ser como auxiliar $^{5}$ (Benzing 1931; Pountain 1985), clasificables estos juntos como verbos de cambio télicos (Mateu Fontanals 2009: 185). Desde el punto de vista semántico y aplicando la teoría sobre los proto-roles temáticos (Dowty 1991), se ha concluido que la resistencia a la sustitución de ser por haber fue mayor en el caso de los verbos cuyo sujeto es prototípicamente paciente (Aranovich 2003: 11). Finalmente, parece que los datos cronológicos del cambio responden a la aplicación en la diacronía de la jerarquía de selección de auxiliares formulada para explicar hechos sincrónicos.

A continuación examinaremos el uso de los auxiliares $a v e r^{6}$ y ser en construcciones intransitivas registradas en la versión sevillana de la Historia del noble Vespasiano, editada en 1499, una obra narrativa difundida y popular en la Europa medieval-, basada en el Evangelium Nicodemi, que cuenta la destrucción de Jerusalén por el emperador Vespasiano presentando los hechos históricos como venganza de la muerte de Jesucristo. Este breve relato debió de tener diferentes versiones manuscritas en las lenguas romances de la península Ibérica, y basadas en estas posteriormente nacerían diversas ediciones impresas, de las que se conocen dos en español (Toledo 1492; Sevilla 1499) y una en portugués (Lisboa 1496). Aunque las versiones paralelas -la edición portuguesa probablemente es una traducción del español (Machado s.f.: 5 y 2010: 126) - se han convertido en objeto de estudios históricocomparativos -por ejemplo Machado (2008), Berta (2014, 2017)-, en este trabajo nos concentraremos en la versión sevillana, que utilizaremos como representante de la situación lingüística de la época preclásica de la historia del español.

Analizaremos los datos recogidos del corpus clasificando los verbos según el auxiliar que seleccionan y distinguiendo los que se documentan

\footnotetext{
${ }^{5}$ Company (1983), al examinar un corpus amplio de textos literarios procedentes del período del siglo XII al XV, ya destaca usos esporádicos de aver en el Poema de mio Cid, y observa el retroceso gradual de ser, que, según su análisis, en La Celestina solo tiene seis ocurrencias con verbos intransitivos. En cuanto a las épocas posteriores, el análisis de García Martín (2001: 117-118) muestra que ser+participio ya es raro a mediados del siglo XVI e Idrisz (2009) no encuentra ningún caso de esta estructura en las Novelas ejemplares de Cervantes. Lapesa (2000: 785) menciona un uso muy tardío, datado en 1836 de era pasada en Larra.
}

${ }^{6}$ A continuación utilizamos la grafía aver, aplicada en el corpus y habitual en la época. 
exclusivamente con aver, los que se registran exclusivamente con ser y los que muestran la alternancia de estos auxiliares. Quedan excluidas -en la medida que sea posible- aquellas construcciones del tipo ser+participio que deben interpretarse como pasivas o reflexivas. Así, no se han tenido en cuenta los ejemplos de voz pasiva ni los de perfecto de verbos pronominales similares a (6a) y (6b), respectivamente. En (6a) aquesta cibdad es paciente de cercada y destruyda, y aparece como sujeto superficial debido a la pasivización. En (6b) fue leuãtado, cuyo sujeto agente es Sant Clemente, corresponde al perfecto del verbo pronominal levantarse, construido con ser, pero generalmente sin pronombre en el español antiguo (Arroyo Vega 2001: 122-165). En casos dudosos hemos utilizado el contexto para desambiguar el significado de la construcción: así en (6c), donde aparece el verbo acabar, susceptible de ser usado como biactancial transitivo o monoactancial inacusativo, el contexto aclara que los valles mencionados fueron acabados por los que labraron, por tanto la construcción debe ser interpretada como pasiva, no como intransitiva inacusativa. Tampoco se han tenido en cuenta las construcciones pronominales auxiliadas con aver -como la que se aduce en (6d)-.

(6) a. aquesta cibdad sera cercada y destruyda y verna a tã grãde destruymiẽto que no quedara piedra sobre ella. (SEV 5582-5584).

b. E quando fue de dia claro Sant Clemente fue leuãtado a fazer oraciõ e vino vna boz del cielo que le dixo: (SEV 5414-5417) ${ }^{7}$.

c. Y enesta manera labrarõ fasta que los valles fuerõ acabados. (SEV 5914-5915).

d. e quãdo se ouierõ recogido ala barca e fuerõ salidos ẽ tierra luego se ẽtro la casa enel abismo cõ Pilatos toda: (SEV 6777-6778).

Durante el análisis contrastaremos nuestros datos con los que proporciona la bibliografía especializada previa. Aunque el análisis que ofrecemos es principalmente descriptivo, a modo de explicación, intentamos aprovechar los resultados de las teorías anteriormente mencionadas. Dedicaremos atención especial a los casos de alternancia donde partiremos de la hipótesis - sustentada por Par (1923: 320) para el catalán antiguo, Yllera (1980: 231-232) para el español antiguo, Grevisse (1969: 605) para el francés- de que la selección de auxiliar es determinada por el carácter accional o resultativo de la construcción,

\footnotetext{
${ }^{7}$ La interpretación reflexiva de este ejemplo es justificada por la forma verbal pronominal simple utilizada en la versión portuguesa: «E tãto que foy dia craro se aleuantou a fazer oraçam: e veo hũa voz do çeeo que lhe disse» (LIS 3677-3679).
} 
el que está vinculado a la agentividad o no agentividad del sujeto. Intentaremos identificar el rasgo [ \pm agentivo] con la ayuda de elementos contextuales, pragmáticos o sintácticos. Sobre la base de Suárez (2016: 158), suponemos que el carácter humano o animado del sujeto implica volición, intencionalidad y control sobre el acontecimiento designado por el verbo, y que el carácter inanimado genera más bien la falta de este control, y que el primer caso implica el empleo de aver, mientras que el segundo el uso de ser. Tal idea es ilustrada por el contraste que se observa entre los ejemplos aducidos en (7) -tomados de Rosemeyer (2014: 185, 165), con sujetos animados-y (8) -tomados de Meilán (1992: 658), con sujetos inanimados-. Basándonos en Rosemeyer (2014: 172) y Suárez (2016: 159) aceptamos que el carácter agentivo del sujeto no solo es derivable de la semántica del verbo o del contexto situacional, sino que puede ser reforzado por la presencia de ciertos elementos -como la de por guarda en (7b) - que aluden a circunstancias como el modo de realizar la acción o la intención de la entidad o persona designada por el sujeto.

a. Salió de las galeas con algunos pocos que con él avían quedado (VIC, 2606)

b. fueron puestos por los campos en la ordenança. E tenía la delantera Traín [...], ca este Traín avía quedado en el real por guarda (CRR, 1379).

(8) a. Esta obra de natura es divina, es a saber intelectual e era solamente quedada en los philosoficos entendimientos en aquella sazon ca de las otras partes poco curavan (DTH 50).

b. Este dañava las pequeñas poblaciones que en calidonia quedadas eran (DTH 110).

Mediante el análisis intentaremos determinar la distribución de los dos auxiliares en cuestión, definir los criterios a los que su selección pudo haber estado sometida en la época de la edición examinada y situar, de esta manera, el texto en este período considerado sumamente importante desde el punto de vista de la historia de la selección de auxiliares en español.

En el corpus analizado fueron registradas en total 62 construcciones formadas por el auxiliar aver o ser y el participio de un verbo intransitivo, entre las cuales hay 22 casos donde el auxiliar es aver y 40 donde esa función es desempeñada por ser. Los verbos cuyos participios coaparecen con estos auxiliares pueden ser divididos en tres grupos: los auxiliados 
exclusivamente con aver, los auxiliados exclusivamente con ser y los que muestran alternancia en la selección del auxiliar.

Los verbos que pertenecen al primer grupo son acahescer, acostumbrar, andar, començar, comer, durar, estar, fablar y trabajar. La mayoría de estos verbos -como andar, començar, comer, fablar y trabajar- no solo es clasificable como intransitivo inergativo -con un sujeto agente caracterizado por el rasgo [+humano]-, sino incluso como transitivo, lo cual explica la auxiliación con aver ilustrada en (9) -según argumentan Yllera (1980: 232) y García Martín (2001: 109).

(9) a. matarõ vn hõbre que luẽgo tiẽpo auia andado asi como loco por la cibdad de Jerusalẽ diziẽdo todos dias (SEV, 5989-5991).

b. e ẽpero no se osauã bautisar fasta que el ẽperador e su fijo Titus ouierõ coméçado (SEV 6523-6524).

c. Y quãdo ouieron comido el enperador los fizo venir ante si e demando les que si creyã en aquel Sãto profeta e ellos dixeron que si. (SEV 5663-5665).

d. emperador e quãdo ouierõ amosados fablado tornaron se a la otra gente alla dõde estaua el mẽsagero del emperador cõ Jacob (SEV 5313-5314).

e. Grãdes gracias do yo a dios e a aquesta sãcta muger que tanto $a$ trabajado por mi. (SEV 5511-5513).

Los verbos andar y començar merecen atención particular. El primero porque, según menciona Yllera (1980: 232), a diferencia de otros verbos de movimiento, se documenta solo con aver $^{8}$, probablemente por usarse también como transitivo. El segundo, porque pertenece a aquellos verbos que muestran variación diatética y pueden usarse como biactanciales transitivos o monoactanciales inacusativos. En el ejemplo citado parece aparecer en una construcción transitiva elíptica, donde el complemento regido ha sido eliminado.

Los verbos acahescer (1), durar (1) y estar -o tal vez, ser ${ }^{9}-$ constituyen un subgrupo interesante por mostrar un comportamiento menos coherente que los anteriores. Aunque el sujeto de los primeros dos suele ser inanimado, mientras que el del último puede ser animado o inanimado indiferentemente, la semántica de los tres verbos le atribuye el proto-rol de paciente, lo que implicaría, según lo expuesto por Aranovich (2003), la selección de ser como auxiliar. Sin embargo, la bibliografía

${ }^{8}$ Tal comportamiento de andar fue confirmado por England (1982: 121-122) en la prosa de Don Juan Manuel.

${ }^{9}$ Arroyo Vega (2001: 94-99), a propósito de la voz pasiva, menciona que en la época aquí examinada estado podía funcionar también como participio de ser. 
especializada solo menciona el uso de este auxiliar a propósito de $a c a(h)$ $e(s) c e r ;$ Yllera (1980: 233) y Elvira (2001: 213) afirman que este verbo en el español medieval normalmente se combinaba con ser, mientras que England (1982: 128) -citado también por Moscoso Mato (2000: 125)— en obras de Don Juan Manuel observa en su caso la alternancia de los dos auxiliares en una distribución equilibrada. Según se ve en (10a), en nuestro corpus la única ocurrencia de este verbo se documenta con aver. Todo esto concuerda con la afirmación de Aranovich (2003: 16), que sitúa la sustitución de ser por aver en el caso de los «verbos dinámicos de existencia y aparición» - entre ellos acaescer- en el siglo XV.

Los otros dos verbos, en cambio, suelen usarse casi exclusivamente con aver. En cuanto a durar, poco examinado por trabajos previos, hay que decir que en el texto analizado se documenta con aver, lo que coincide con los datos de England (1982: 125), quien atribuye la preferencia firme por este auxiliar a la coaparición frecuente del verbo en cuestión con elementos adverbiales de tiempo similares a complementos directos como el constituyente ocho dias destacado en negrita en (10b) - ${ }^{10}$. Esta argumentación es apoyada por Cano Aguilar (1987: 321-322), quien en cuanto al español actual, confirma que «los complementos de 'extensión' temporal o 'duración'», «pueden convertirse también en objetos directos». Estos hechos pueden atribuir a durar cierto nivel de transitividad ${ }^{11}$. El verbo estar/ser -a diferencia de otras lenguas- se auxilia en el corpus con aver; ello coincide con el uso medieval habitual (Yllera 1980: 237; García Martín 2001: 110-111; Octavio de Toledo 2002: 259), salvo casos esporádicos de ser+estado atribuidos generalmente a la influencia aragonesa, catalana o italiana (Yllera 1980: 229, 237; Lapesa 2000: 784). Desde el punto de vista de la cuestión de uso del auxiliar es importante resaltar que tanto Yllera (1980: 237) como García Martín (2001: 110) mencionan que hasta el siglo XIII ser y estar no contaban con formas compuestas en absoluto. Nótese, además, que las estructuras formadas con el participio estado -de modo similar a durado - también muestran coocurrencia frecuente con sintagmas adverbiales de tiempo -como ocho dias, tres días, tãto tiẽpo y dos años, destacados en negrita, en (10c-f)-: esta construcción aparece siete veces en el corpus analizado acompañada de complementos de tiempo similares.

${ }^{10}$ En este sentido durar podría ser incluido entre los verbos que se construían con complementos de medida, siempre auxiliados con aver, según dice Octavio de Toledo (2002: 259).

${ }^{11}$ Nótese que según Sorace (2000: 867-868) en el italiano el verbo durare muestra alternancia de auxiliares siendo essere el auxiliar preferido por el estándar. 
(10) a. e cõtar vos he vna grãd marauilla que enesta cibdad a acahescido. (SEV 6162-6163)

b. Y quãdo esta fiesta ouo durado ocho dias ayũtarõ se todos los caualleros e nobles dela cibdad enel palacio del ẽperador (SEV 6605-6607).

c. e quãdo el ẽperador ouo estado ocho dias Sãt Clemeĩte le fue a ver e dixo le: (SEV 6525).

d. Quãdo el emperador ouo estado tres dias ẽ Acre partio cõ toda su gẽte todo quãto auia ganado en Jerusalen (SEV 6436-6437).

e. mas a el no le semejo que y ouiese estado tãto tiẽpo como desdel viernes que descẽdio dela cruz el cuerpo de Jesu Christo fasta el domĩgo que dixerõ que Jesu Christo era resuscitado (SEV 6476-6481).

f. El mal hõbre que tanto de estrecha mente auia estado dos años ẽla prisiõ del pozo (SEV 6795-6796).

El verbo acostumbrar (1) aparece en el corpus auxiliado con aver, pero también era posible su uso con ser -cfr. (11a), de nuestro corpus y (11b), procedente de otro texto-. Sin embargo, probablemente es importante que cuando se utiliza en una perífrasis -como en los ejemplos aducidos- la selección del auxiliar parece depender de las características semánticas del verbo en infinitivo -cfr. fazer en (11a) culpar en (11b)-.

(11) a. Señor todos somos aparejados de fazer quãto podamos e avemos acostũbrado de fazer. (SEV 5946-5948).

b. Et el rey le respondio que don Iohan Nunyez sabie que el no era acostumbrado de culpar a el ni a otro ninguno sin merecerlo (GCE fol. 143r)

Resumiendo lo expuesto hasta ahora, en nuestro corpus todos los verbos arriba enumerados se documentan únicamente con el auxiliar aver.

Al segundo grupo pertenecen los verbos acabar, arribar, entrar, escapar, yr, llegar, nascer, morir, resuscitar y passar, documentados solo con el auxiliar ser. Entre estos casos acabar (1), escapar (1), resuscitar (2) y pasar (1) son verbos que expresan movimiento inherentemente dirigido o cambio de estado y presentan la alternancia diatética mencionada más arriba (Elvira 2001 y Rodríguez Molina 2006), pudiendo usarse como biactanciales transitivos o monoactanciales inacusativos. Una consecuencia de esta duplicidad de estructuras es que en estos casos a veces resulta difícil juzgar si la construcción con ser es pasiva o intransitiva (Elvira 2001: 225-234; Romani 2008), lo que hace necesario examinar el contexto situacional y sintáctico para poder determinar la naturaleza de cada estructura. 
(12) a. e quãdo ouo acabado su sermõ e el oficio fue acabado e la missa fue dicho [sic!] el emperador mãdo que (SEV 6600-6603).

b. ca muchos fuerã escapados a vida e por aquesta razõ murierõ (SEV 6370).

c. despues resuscito al tercero dia e a los quarenta dias se subio a los cielos despues que fue resuscitado ẽ presencia delos sus // [...] (SEV 5376-5378).

d. El sol puesto comẽço a salir luego ẽ horiẽte biẽ asi como si la noche fuese pasada fue dia claro. (SEV 5973)

En (12a) la coocurrencia con la estructura transitiva ouo acabado su sermõ y la coordinación con la construcción evidentemente pasiva la missa fue dicho nos hacen atribuir una interpretación más bien pasiva a fue acabado. En (12b), en cambio, el contexto situacional sugiere que el sujeto muchos no es paciente de un verbo biactancial pasivizado con agente por referirse a personas que habrían quedado vivas si ellas mismas hubiesen actuado de una manera diferente. En (12c), la construcción fue resuscitado tiene como antecedente equivalente la forma simple intransitiva resuscito, por tanto la interpretación pasiva también aquí puede ser excluida. Finalmente, en (12d) el sintagma la noche no es susceptible de aparecer como paciente en una estructura biactancial, lo que igualmente excluye la interpretación pasiva. Consideramos, por tanto, justificada la interpretación intransitiva en (12b-d). Nótese que los sujetos de las construcciones aducidas en (12b-c) designan a personas capaces de actuar, pero sin desempeñar el proto-rol de agente, así como en (12d) el sujeto inanimado es la noche.

Los verbos arribar (1), entrar (2), yr (1), llegar (5) y tornar (2) pertenecen a la clase de verbos de movimiento inherentemente dirigido, situados entre los últimos en perder la posibilidad de seleccionar ser como auxiliar. Aunque en el caso de arribar y tornar Benzing sitúa la última documentación con ser en fechas relativamente antiguas -con datación del siglo XIV y XV, respectivamente-, esto probablemente se debe a que estos verbos cayeron en desuso. A pesar de esta resistencia a la generalización de aver, según Yllera (1980: 234-235) y Suárez (2016: 158 ) en el español antiguo esta clase de verbos también aceptaba dicho auxiliar, hecho demostrado por la alternancia de auxiliares del tipo era llegado llegado ha y entrados son an entrado ilustrada en los ejemplos aducidos en (5). Tal variación puede ser explicada con la hipótesis de López García (2016), que atribuye a verbos de movimiento como llegar dos maneras de comportarse: la de verbo de 'cambio de estado', que selecciona un sujeto agente que realiza un desplazamiento con una 
trayectoria y la de verbo de 'aparición', que selecciona un sujeto tema que experimenta la acción. En nuestro corpus, sin embargo, se documenta exclusivamente la auxiliación con ser, ilustrada en (13).

(13) a. despues que partierõ de Acre el emperador ni otra persona nũca supierõ dellos cosa nigũa fasta que fuerõ arribados cada vno ẽ su tierra (SEV 6456-6460).

b. e acosto se al lugar por el qual aquel jnfante era entrado (SEV 5391).

c. e no hallo lugar ni señal por donde aquel jnfante fuesse étrado (SEV 5392-5393).

d. quando // fueron entrados ẽla cibdad los caualleros que trayan el preso quisieron saber qual era el alguazil de todos los dela cibdad (SEV 6716-6719).

e. Mas por quãto la mayor parte dela gente era yda a celebrar la fiesta ẽ Jerusalẽ estauan enel castillo muy pocos e quisierõ se dar mas el enperador no los quiso tomar a merced (SEV 5618-5619).

f. quãdo fuerõ llegados fueron a ver al emperador a los palacios donde estaua e fizierõ le reuerẽcia (SEV 5144-5145).

g. e quãdo Gays el senescal fue llegado al lugar fizo armar vna fusta para passar ẽ Jherusalem: (5211-5212).

h. Y quãdo fuerõ llegados ẽ Acre estouierõ ay tres dias (SEV 6416-6417).

i. el segũdo dia que Gays el senescal fue llegado a Roma auia de ser Titus coronado emperador. (SEV 5354-5355).

j. En aquella sazõ Gays su senescal fue // llegado a Roma: el emperador auia fecho juntar sus cortes de todos los nobles de su jmperio (SEV 5350-5351).

k. prometistes que quando seriades tornado de Jerusalẽ e ouiesedes tomado vẽgãça dela su muerte si a el plazia que tornasedes que luego vos bautizariades (SEV 6537-6543).

l. E quãdo Gays el senescal fue tornado de Jerusalem ẽ Roma con la sancta Veronica que cõ ella traya el sancto paño dõde era la faz de Jesu Christo nuestro redẽtor. (SEV 5343-5346).

En los ejemplos arriba citados los verbos en cuestión aparecen casi exclusivamente en subordinaciones adverbiales de tiempo, introducidos en la mayoría de los casos por quando -en (13d-h)-,y en el resto por otros elementos referentes al tiempo, como fasta que -en (13a)-, el segundo dia que -en (13i) - y en aquella sazõ -en (13j), donde probablemente falta la conjunción quando-. En estos casos es posible atribuir al sujeto superficial el papel de experimentador que aparece en algún lugar y considerar el evento descrito en la subordinación -la aparición o presencia de la persona designada por el constituyente sujeto- como una 
circunstancia de fondo para la acción mencionada en la parte principal de la oración. En (13b-c), en cambio, las construcciones verbales era entrado y fuesse étrado se encuentran en subordinaciones de relativo referentes a lugar, y el sujeto superficial aquel jnfante parece designar un agente que supuestamente fue capaz de controlar la acción de 'entrar'. Asimismo, en (13e) la construcción erayda es acompañada por el constituyenteadverbial final a celebrar la fiesta ẽ Jerusalẽ, lo que -de acuerdo con Rosemeyer (2014: 51) - implica una interpretación agentiva, no resultativa. Nótese que López García solo atribuye comportamiento doble -inacusativa e inergativa- a llegar y no a otros verbos de movimiento.

Los verbos morir (4) y nascer (1) también pertenecen a los verbos que -según vemos en (14)- en nuestro corpus se documentan exclusivamente con ser. Tal hecho no sorprende teniendo presente que varios autores los mencionan entre los más resistentes al uso de aver (Benzing 1931; Yllera 1980: 234; Aranovich 2003: 57). Su resistencia ante la presión de la difusión de aver puede deberse a su clasificación como verbos de 'cambio de estado' y al carácter prototípicamente no agentivo de su sujeto (Aranovich 2003: 14). Conviene destacar, sin embargo, la divergencia que los dos verbos muestran en la evolución de la selección de auxiliar: a diferencia de morir, nascer se documenta con aver desde muy antiguo y parece aceptar definitivamente este auxiliar más temprano. La diferencia puede estar relacionada con el hecho de que muerto era un participio compartido por el intransitivo morir y por el transitivo matar, por lo cual algunas veces se neutralizaba la diferencia entre el valor de perfectiva y de pasiva en la construcción ser+muerto (England 1982: 127; Elvira 2001: 217, 235). Tal confusión se observa en el ejemplo (14a), procedente de nuestro corpus, donde la construcción fue muerto se documenta dos veces. En la primera ocurrencia es probable la interpretación pasiva -es decir, el significado de 'fue matado'-, debido a que se encuentra coordinada con una construcción evidentemente pasiva -fue juzgado a grão tuerto por Pilatos-, pero en la segunda está vinculada con la construcción resuscito al tercero dia, donde se crea una oposición más bien entre los significados de los intransitivos inacusativos resuscitar y morir.

También ha sido registrada otra ambigüedad, vinculada con la polivalencia funcional de ser, mencionada por Romani (2008: 1078-1079); a saber, en la Edad Media, fuera de las construcciones resultativas intransitivas y pasivas, ser también podía aparecer en estructuras atributivas, ejerciendo funciones hoy reservadas para estar. Así, en (14b) la construcción era muerto puede ser interpretada como 
intransitiva de anterioridad - con el significado de 'había muerto'- o como atributiva de simultaneidad - con la acepción de 'estaba muerto'-. La misma ambivalencia se observa en (14e) con la estructura era nascido. La ambigüedad se evita, sin embargo, en (14c-d), donde los elementos adverbiales por que y de tal guisa aluden al motivo o al proceso del fallecimiento y no a su consecuencia.

(14) a. Si aquel santo profeta que fue muerto ẽ Jerusalẽ e fue juzgado a grão tuerto por Pilatos mi adelantado a requirimiẽto delos judios e resuscito al tercero dia que fue muerto me da salude yo tomare vẽgãça delos falsos judios que lo mataron luego que sea sano. (SEV 5402-5407).

b. E quãdo Pilatos vido al Rey Archileus que era muerto fue muy triste e ayrado e entro se ẽ la cibdad (SEV 6232-6233).

c. e deziã todos a vna boz que aquel que todos dias dezia ven Vaspasiano ẽ Jerusalẽ es muerto por que nos creemos que fuese profeta cõtra nos. (SEV 6012-6014).

d. quãdo el enperador ouo leydas las cartas e supo que de tal guisa Pilatos era muerto fizo se mucho marauillado (SEV 6785-6787).

e. ca los sus sabios le dixerõ que el rey delos judios era nascido delo qual ouo muy grãde pesar (SEV 6209-6212).

El tercer grupo, muy reducido en comparación con los anteriores, es formado por los verbos quedar (2), salir (7) y venir (15), cuyos participios se documentan tanto con aver como con ser en la función de verbo auxiliar. Esta alternancia pertenece a aquel tipo de variación donde según Rodríguez Molina (2006: 1069) «la diferencia del auxiliar no conlleva un cambio en la diátesis de la cláusula ni en el significado del verbo». Como ya se ha señalado, para algunos autores esta alternancia es libre, mientras que otros creen que la selección del auxiliar obedece a ciertas condiciones, cuestión que examinamos a continuación mediante el análisis semántico-pragmático de las construcciones recogidas.

En cuanto al verbo quedar, varían las opiniones acerca de si suele construirse con ser (Elvira 2001: 220) o con aver (Yllera 1980: 235), pero según algunos desde el siglo XV se asocia exclusivamente con este último (Aranovich 2003: 5, basado en Benzing 1931). Los ejemplos medievales citados en (15) -aducidos originariamente por Meilán (1992: 658) y Rosemeyer (2014: 185, 165), que repetimos aquí- muestran, en cambio, más bien la selección de ser cuando lo designado por el sujeto es inanimado y la de aver cuando es animado y susceptible de controlar la situación actuando como agente. 
(15) a. Esta obra de natura es divina, es a saber intelectual e era solamente quedada en los philosoficos entendimientos en aquella sazon ca de las otras partes poco curavan (DTH 50).

b. Este dañava las pequeñas poblaciones que en calidonia quedadas eran (DTH 110).

c. fueron puestos por los campos en la ordenança. E tenía la delantera Traín [...], ca este Traín avía quedado en el real por guarda (CRR, 1379).

d. Salió de las galeas con algunos pocos que con él avían quedado (VIC, 2606)

En nuestro corpus las dos ocurrencias de quedar se reparten entre los dos auxiliares, tal como se puede observar en (16).

(16) a. e estos judios que son quedados sean para mi e guardad los biẽ. (SEV 6385-6386).

b. ca ẽ toda esta tierra no ha quedado judio que algo valga que no sea aqui venido por honrrar la fiesta muy marauillosamẽte (SEV 5896-5897).

La distribución de los auxiliares parece corresponder, al menos parcialmente, a la misma que ha sido observada en (15), aunque aquí los sujetos en ambos casos contienen el rasgo [+humano]. En (16a) la estructura estos judios que son quedados se refiere a los judíos que no fueron vendidos por los romanos vencedores después de la ocupación de Jerusalén, por tanto, no se les puede atribuir agentividad alguna. En (16b), en cambio, la situación no es tan clara. Por una parte, la expresión no ha quedado judio que algo valga alude a aquellos judíos que no fueron a la ciudad a participar en las actividades festivas, es decir, que se han quedado en casa intencionadamente, lo cual supone cierto grado de agentividad y control sobre la situación. Por otra parte, la misma expresión también puede ser interpretada como 'no hay aquí ningún judío que algo valga', lo que no supone control sobre la situación.

A diferencia de quedar, apenas registrado en el corpus analizado, salir y venir, los otros dos verbos que muestran alternancia de auxiliares, muestran un uso bastante frecuente: el primero tiene 7, el segundo 15 ocurrencias, entre las cuales cada uno cuenta con un solo caso de auxiliación con aver. La predominancia de ser no sorprende si tenemos presente que estos verbos suelen ser categorizados entre los últimos en perder cronológicamente la posibilidad de aceptar este auxiliar.

En cuanto a salir, podemos observar que en su caso algunas construcciones formadas con ser pueden ser interpretadas como resultativas con sujetos no agentivos, especialmente cuando este tiene el 
rasgo [-humano]. Así, en (17a-b) los sintagmas el sol y aquel cõsejo, por designar entidades no personales, son sujetos no agentivos de fue salido y era salido, respectivamente. En (17c), aunque el sujeto aparente las gẽtes es caracterizado por el rasgo [+humano], en vez de agente, es más bien beneficiario de actos realizados previamente por otras figuras: los nobles que habían acompañado al emperador en el sitio de Jerusalén, ya bautizados, al regresar a sus tierras hicieron convertir a sus pueblos al cristianismo, cuya consecuencia es que estos fueron salvados, es decir, fuerõ salidas del error delos diablos. En (17d) la construcción fuerõ salidos aparece en una subordinación temporal introducida por quãdo y parece describir una circunstancia que sirve de fondo para el evento descrito por la parte principal de la oración, pero en (17e) fue salida aparece en oración principal y parece referirse a una acción controlada por el sujeto Veronica. En (17f), donde también encontramos el uso de ser como auxiliar, la agentividad del sujeto es reforzada por elementos contextuales: la presencia de la construcción final para pelear cõ nosotros es incompatible con un sujeto no agentivo, puesto que supone intencionalidad de parte de este. Este ejemplo demuestra, de acuerdo con Suárez (2016: 159), «que la presencia de ser + participio no conlleva automáticamente lectura no agentiva». En (17g) se documenta la única ocurrencia de aver con el participio salido, donde el sujeto - ausente en la estructura superficial-, se entiende como agente que controló la acción descrita por el complejo verbal avia salido, teniendo en cuenta que consiguió escaparse de la cárcel. La idea de acción es reforzada por la presencia del interrogativo como, que señala que la proposición interrogativa subordinada se refiere a la manera de escaparse.

(17) a. Quando el dia fue claro e el sol fue salido el ẽperador no quiso adorar los ydolos como solia por quãto no auia enellos firme creencia por las palabras que su senescal le auia dicho. (SEV 5448-5449).

b. ouierõ muy grãde pesar e dixerõ entre si que aquel cõsejo era salido de la cabeça de Jafel. (SEV 5817-5819)

c. e las gẽtes fuerõ salidas del error delos diablos ẽ que creyã (SEV 6628-6630).

d. e quãdo se ouierõ recogido ala barca e fuerõ salidos ẽ tierra luego se ẽtro la casa enel abismo cõ Pilatos toda: (SEV 6777-6778).

e. e fue se a la puerta del senescal e fue salida Veronica de fuera dela puerta e fallo ay al sãto hõbre e dixo le: (SEV 5420).

f. Amigos sabed que Pilatos cõ toda la gẽte dela cibdad es salido para pelear cõ nosotros por que es menester que sabia mẽte salgamos a el al canpo: (SEV 5942-5945). 
g. E despues demãdo le como auia salido de la cibdad que el avia oydo dezir que Pilatos lo auia puesto ẽ prision (SEV 5880-5882).

Por lo que se refiere a venir, este verbo se documenta con una absoluta predominancia del auxiliar ser; en algunos casos esta estructura parece expresar el resultado de una acción, como ocurre en (18a-c).

(18) a. Quando el emperador oyo dezir que Gays su senescal era venida [sic!] ouo muy grande gozo e plazer e deseaua mucho fablar con el por la su salud. (SEV 5348-5350).

b. quãdo el alguazil fue venido cõ otros hõbres buenos dela cibdad fizo poner a Pilatos ẽla barca (SEV 6772-6773).

c. e el ẽperador mãdo que fiziesen venir los senadores de Roma e quãdo fuerõ venidos el emperador les dixo: (SEV 6649-6652).

d. Grãde fue la fambre e la careza que era ẽ la cibdad por razõ delas muchas gẽtes que estauã aqui que erã venidas a hõrrar la fiesta e no auian traydo viãdas (SEV 6044-6046).

e. e yo so aqui venida por guarescer al Emperador segũd que yo guaresci por virtud del sãto profeta Jesu Christo (SEV 5429-5431).

f. Y segũd que yo pienso vos sodes venido por vengar la muerte del sãto profeta que a gran tuerto tomo muerte e pasion ẽ Jherusalẽ (SEV 5650-5653).

g. porque vos ruego que me digades dõde sodes e como vos dizẽ e por que causa sodes venido a esta cibdad (SEV5228-5230).

h. por que sepades que yo soy venido enesta tierra que si por a ventura podria hallar aqui algũas cosas que fuessen prouechosas a mi señor para lo sanar de aquella fuerte dolẽcia (SEV 5242-5245).

i. E quãdo Veronica fue delãte el senescal, Jacob le dixo la razon por que el senescal era alli venido: (SEV 5284-5286).

j. De como llego Gays en Jherusalẽ e de como lo acogio Jacob en su casa e de como dixo el senescal Gays a Jacob el porque avia venido a Jherusalem. (SEV 5218-5219).

En (18a) la construcción era venida -que debe ser leída como era venido- alude a la presencia del sujeto representado por el sintagma Gays, su senescal en Roma, o sea, a una situación y no a la acción de desplazarse; en (18b-c), de modo similar, las construcciones fue venido y fuerõ venidos más bien aluden a la presencia del alguazil y de los senadores, respectivamente, como consecuencia de su llegada, mientras que el acto que narra cada una de esas oraciones es representado por fizo poner a Pilatos ẽla barca, de un lado, y el emperador les dixo de otro. En numerosos casos, sin embargo, que ilustramos en (18d-i), la presencia de elementos 
adverbiales finales o causales -que aquí destacamos en negrita-, demuestran la intencionalidad de la acción designada por el verbo y el control de la entidad designada por el sujeto. Estos ejemplos, junto con los que aducimos en (19), citados por estudios previos, de acuerdo con Rosemeyer (2014: 149) y Suárez (2016: 159), contradicen la suposición de que la construcción ser+participio sea necesariamente resultativa.

(19) a. E demandaron le porque era alli uenido de tan luenga tierra (EdE E: 514, 14. (174r), citado también por Stolova (2016: 313)

b. que era ydo a valladolid a descabeçar algunos grandes de castilla (ATA 2025, citado por Rosemeyer 2014: 149)

c. Dyxo l'que tras el puerco era aý venido (LFG p. 218, citado por Suárez 2016: 159)

d. Et agora es venido con xxix Reyes por vengar a su hermano por conbrar Valencia (EdE E $E_{2}$ :943, 21, (229r), citado también por Suárez ibídem)

Lo que conviene destacar es que en el único ejemplo registrado con aver, que citamos en (18j) el sujeto -el senescal llamado Gays-, se presenta como agente puesto que se trata de que este se había desplazado a Jerusalén y no de que se encontraba ya allí, y la expresión el porque alude a las razones que lo motivaron a realizar tal viaje.

El análisis de los casos de alternancia documentada con quedar, salir y venir muestra que mientras que ser se registra en construcciones con función tanto eventual como resultativa, con indiferencia en cuanto al carácter agentivo o no agentivo del sujeto, el uso de aver se limita a las construcciones eventuales donde el contexto nos hace atribuir el papel de agente al sujeto.

Después de analizar los datos del corpus podemos llegar a las conclusiones siguientes. En la edición sevillana de la Historia del noble Vespasiano observamos la alternancia de auxiliares que caracterizó el español medieval y que sobrevivió aproximadamente hasta el siglo XVII, por tanto, en este sentido, el texto, procedente de finales del siglo XV, parece corresponder a las expectativas. Una parte considerable de verbos se documenta únicamente en construcciones del tipo aver + participio. La mayoría de este grupo normalmente se asocia con un sujeto agente y puede ser utilizado también como transitivo, factores que explican el empleo de este auxiliar durante toda la Edad Media y, como consecuencia, también en nuestro corpus. No es este el caso de acahescer y estar -o ser-, recogidos exclusivamente con aver, pero que no forman parte del grupo mayoritario mencionado, por no tener sujeto agente niaplicación transitiva 
verdadera. En cuanto a aca(h)escer, debemos recordar que pertenece a la clase de verbos dinámicos de aparición, auxiliados originariamente con ser, que, como tal, para la época en cuestión ya habría sido afectado por un cambio en la selección de auxiliar. El verbo estar -o ser-, a su vez, pertenecería a los verbos estáticos de existencia y aparición, que fueron los primeros en perder la auxiliación con ser, por tanto este cambio ocurriría en una época cuando el verbo en cuestión aún no contaba con una forma compuesta. Otro grupo importante -y bastante heterogéneode verbos documentados en construcciones formadas con participio se ha registrado exclusivamente con ser, generalmente en construcciones que aluden al resultado de una acción o acontecimiento. Entre ellos algunos pertenecen a los que muestran alternancia con variación diatética entre el uso transitivo y el inacusativo, y cuando aparecen en esta segunda función, seleccionan ser. En este grupo se encuentran verbos de movimiento inherentemente dirigido y verbos de cambio de estado. Otros dos grupos son formados por verbos de movimiento inherentemente dirigido y por verbos que expresan cambio de estado, pero que no muestran alteración diatética. Estos dos grupos han sido clasificados como los más resistentes a la difusión de aver, por tanto no sorprende que en nuestro texto de finales del siglo XV se hayan registrado con el auxiliar ser. Solamente los verbos quedar, salir y venir muestran alternancia en la selección de auxiliares sin alteración diatética. Con este comportamiento quedar contradice las expectativas, debido a que según la bibliografía especializada en la época del nacimiento del texto este verbo se auxiliaba únicamente con aver. La selección del auxiliar -de acuerdo con nuestra hipótesis- parece obedecer al carácter agentivo o no agentivo del sujeto y a la naturaleza eventual o resultativa de la predicación, pero conviene admitir que la muy baja ocurrencia de este verbo no nos permite extraer conclusiones definitivas referentes a la sistematicidad de esta distribución aparentemente funcional. En el caso de los otros dos verbos -mucho más documentados- que muestran alternancia se debe decir que esta está en consonancia con el comportamiento esperado en base a los resultados de los estudios previos. Conviene añadir, sin embargo, que en su caso el uso de aver es muy minoritario, lo que concuerda con el hecho de que estos, como verbos de movimiento, fueron más resistentes a la difusión de aver y en el período examinado se usarían más con ser. En cuanto a la distribución de los dos auxilares podemos hacer constar que la estructura ser + participio aparece tanto con sujeto agentivo como con sujeto no agentivo y tanto con valor eventual como con función resultativa, mientras que los escasos ejemplos de aver + participio se documentan 
en construcciones que aluden a eventos intencionales controlados por un sujeto agentivo. Ello puede demostrar que el avance de aver en la función de auxiliar comenzaría en este tipo de enunciados entrando una verdadera alternancia libre con ser limitada a este contexto.

\section{REFERENCIAS BIBLIOGRÁFICAS}

\section{Corpus analizado}

$\mathrm{SEV}=$ Ystoria del noble Vespesiano emperador de Roma (Sevilla 1499). En José Barbosa Machado, História do mui Nobre Vespasiano Imperador de Roma. Edição e estudo. Braga: Edições Vercial, 2010. Kindle Version.

\section{Otras fuentes citadas}

ALE=Libro de Alexandre. Ed. de Jesús Cañas, Madrid: Cátedra, 1988. (CORDE)

APO=Libro de Apolonio. Ed. de Dolores Corbella, Madrid: Cátedra, 1999.

ARM=Auto de los Reyes Magos. Edición digital a partir de la edición de Ramón Menéndez Pidal, Textos medievales españoles. Madrid: Espasa-Calpe, 1976: 171-177. Alicante: Biblioteca Virtual Miguel de Cervantes, 1999.

ATA=Alfonso Martínez de Toledo, Atalaya de las corónicas. Ed. de James B. Larkin, Madison: Hispanic Seminary of Medieval Studies, 1983.

CEM=Ramón Menéndez Pidal, Crestomatía del español medieval. Tomo I. Madrid: Gredos, 1982.

CORDE=Real Academia Española, Banco de datos (CORDE) [en línea]. Corpus diacrónico del español. [http://www.rae.es]

CRR=Pedro de Corral, Crónica del rey don Rodrigo, postrimero rey de los godos (Crónica sarracina). Ed. de James Donald Fogelquist. Madrid: Castalia, 2001.

DTH=Los doze trabajos de Hércules. Ed. de Margherita Morreale, Madrid: Real Academia Española, 1958.

ECL=Don Juan Manuel, El conde Lucanor. Ed. de Guillermo Serés, Barcelona: Crítica, 1994.

EdE=Alfonso X, Estoria de España. Según Aengus Ward (ed.), Estoria de Espanna Digital v.1.0, Birmingham: University of Birmingham, 2016, [estoria.bham.ac.uk] Web. 19/10/2019. 
GCE=Juan Fernández de Heredia, Gran crónica de España. III. BNM, ms. 10134. Ed. de Juan Manuel Cacho Blecua, Zaragoza: Universidad de Zaragoza, 2003. (CORDE)

LFG=Libro de Fernán González. Ed. de Itzíar López Guil, Madrid: Biblioteca Nueva, 2001. (CORDE)

LIS=Estoria de muy Nobre Vespesiano Emperador de Roma (Lisboa 1496).

En José Barbosa Machado, História do mui Nobre Vespasiano Imperador de Roma. Edição e estudo. Braga: Edições Vercial, 2010. Kindle Version.

PMC=Poema de Mio Cid. Reproducción digital de la edición paleográfica por D. Ramón Menéndez Pidal, Madrid, [s.n.], 1961, Alicante: Biblioteca Virtual Miguel de Cervantes / Madrid: Biblioteca Nacional, 2002. VIC=Gutierre Díez de Games, El victorial. Ed. de Rafael Beltrán Llavador, Madrid: Taurus, 1994.

\section{Obras consultadas}

Andrés Suárez 1994: Irene Andrés Suárez. El verbo español. Sistemas medievales y sistema clásico. Madrid: Gredos.

Aranovich 2003: Raul Aranovich. «The semantics of auxiliary selection in Old Spanish». Studies in Language, 27/1: 1-37. Web. 10/11/2019.

Arias Álvarez 2000: Beatriz Arias Álvarez. «'Ser' o 'haber' (+ participio): divergencia hispánica». Revista Española de Lingüística Aplicada, Volumen extra 1: 193-213. Web. 10/11/2019.

Arroyo Vega 2001: Paloma Arroyo Vega. La diátesis verbal en el castellano del siglo XV. Cuadernos de Filología. Anejo XXXIX. Valencia: Universitat de València.

Batlle et al 2016: Mar Batlle et al. Gramàtica histórica de la llengua catalana. Barcelona: Publicacions de l'Abadia de Montserrat.

Benzing 1931: Joseph Benzing. «Zur Geschichte von ser als Hilfszeitwort bei den intransitiven Verben im Spanischen». Zeitschrift für romanische Philologie, 60: 385-460.

Berta 2014: Tibor Berta. «Os tempos verbais compostos nas versões castelhana e portuguesa da História do Mui Nobre Vespasiano Imperador de Roma». Bartha-Kovács Katalin et al. (eds.), „Transfert nec mergitur". Albert Sándor 65. születésnapjának tiszteletére. Szeged: JatePress, 141-151.

Berta 2017: Tibor Berta. «La influencia española en la versión portuguesa de la Historia de Vespasiano. Los tiempos compuestos». Ljiljana Marković \& Augusta Consorti (eds.), Identidad, movilidad y 
perspectivas en los estudios de cultura. Belgrado: Universidad de Belgrado, 93-128.

Burzio 1986: Luigi Burzio. Italian syntax: A Government-Binding approach. Dordrecht-Boston: Reidel Pub. Co.

Cano Aguilar 1987: Rafael Cano Aguilar. Estructuras sintácticas transitivas en el español actual. Madrid: Gredos.

Cennamo 2008: Michela Cennamo. "The rise and development of analytic perfects in Italo-Romance». Thorhallur Eythórsson (ed.), Grammatical Change and Linguistic Theory: the Rosendal Papers. Amsterdam: Benjamins, 115-143.

Company 1983: Concepción Company. «Sintaxis y valores de los tiempos compuestos en el español medieval». Nueva Revista de Filología Hispánica, 32/2: 235-257. Web. 10/11/2019.

Dowty 1991: David Dowty. «Thematic Proto-Roles and Argument Selection». Language, 67/3: 547-619.

Eberenz 1991: Rolf Eberenz. «Castellano antiguo y español moderno: reflexiones sobre la periodización en la historia de la lengua». Revista de filología española, 71: 79-106.

Eberenz 2009: Rolf Eberenz. «La periodización de la historia morfosintáctica del español: propuestas y aportaciones recientes». Cahiers d'études hispaniques médiévales 32: 181-201. Web. $10 / 11 / 2019$.

Elvira 2001: Javier Elvira. «Intransitividad escindida en español: El uso auxiliar de ser en español medieval». Estudios de Lingüística, Universidad de Alicante, 15: 201-245.

England 1982: John England. «Ser and aver with the past participles of intransitive verbs in the works of Don Juan Manuel». Don Juan Manuel: VII centenario. Murcia: Universidad de Murcia/Academia Alfonso X el Sabio, 117-133.

García Martín 2001: José María García Martín. La formación de los tiempos compuestos del verbo en español medieval y clásico. Aspectos fonológicos, morfológicos y sintácticos. Valencia: Universitat de València.

Grevisse 1969: Maurice Grevisse. Le bon usage: grammaire francaise avec des remarques sur la langue francaise d'aujourd'hui. Gembloux: Éditions Duculot.

Idrisz 2009: Ágnes Idrisz. «El comportamiento de los verbos haber y ser en función de auxiliar de los tiempos compuestos (siglos XIIIXVII)», Acta hispanica, 14: 99-112. 
Keniston 1937: Hayward Keniston. The syntax of Castilian prose. The sixteenth century. Chicago: The University of Chicago Press.

Lapesa 1988: Rafael Lapesa. Historia de la lengua española. Madrid: Gredos.

Lapesa 2000: Rafael Lapesa. Estudios de morfosintaxis histórica del español. Madrid: Gredos.

López García 2016: Fernando López García. «¿Dos verbos llegar en español?». Estudios de Lingüística, Universidad de Alicante, 30: 16-180. Web. 10/11/2019.

Machado 2008: José Barbosa Machado. «Alguns conectores e marcadores de discurso em três versões da 'História do Mui Nobre Vespasiano Imperador de Roma'». Estudos Linguísticos/Linguistic Studies, 2: 99-122. Web. 09/05/2016.

Machado 2010: José Barbosa Machado (ed.). História do mui Nobre Vespasiano Imperador de Roma. Edição e estudo. Braga: Edições Vercial.

Machado (s.f.): José Barbosa Machado. «Press and translation as Changing Factors in the 15th century portuguese language and culture». Web. 29/05/2016.

[http://alfarrabio.di.uminho.pt/vercial/zips/machad13.pdf]

Mateu Fontanals 2005: Jaume Mateu Fontanals. «La selecció de l'auxiliar en els verbs intransitius del català antic: alguns aspectes descriptius i explicatius». Caplletra, 38: 211-240.

Mateu Fontanals 2009: Jaume Mateu Fontanals. «Gradience and auxiliary selection in Old Catalan and Old Spanish». Paola Crisma \& Giuseppe Longobardi (eds.), Historical Syntax and Linguistic Theory. Oxford: Oxford University Press, 176-193.

Meilán García 1992: Antonio Meilán García. «El verbo ser como auxiliar en la prosa (pre)renacentista». Manuel Ariza et al. (eds.), Actas del II congreso internacional de historia de la lengua española. Madrid: Pabellón de España, 653-663. Biblioteca Virtual Miguel de Cervantes. Web. 10/11/2019.

Mithun 1991: Marianne Mithun. «Active/Agentive case marking and its motivations». Language, 67/3 (1991): 510-546.

Molho 1975: Mauricio Molho. Sistemática del verbo español: aspectos, modos, tiempos. Madrid: Gredos.

Moll 2006: Francesc de B. Moll. Gramàtica histórica catalana. València: Universitat de València.

Moscoso Mato 2000: Eduardo Moscoso Mato. Os tempos compostos no galego medieval. Anuario Galego de Filoloxia. Anexo 46. Santiago de Compostela: Universidade de Santiago de Compostela. 
Octavio de Toledo y Huerta 2002: Álvaro Octavio de Toledo y Huerta. «Auxiliación con ser de verbos intransitivos de movimiento (14501600): el caso de ir(se)». Res Diachronicae, 1: 257-269.

Oudin 1639: César Oudin. Grammaire espagnolle mise et expliquée en françois. Bruxelles: Jean Mommart.

Par 1923: Anfòs Par. Sintaxi catalana segons los escrits en prosa de Bernat Metge (1398). Halle (Sale): Verlag von Max Niemeyer.

Penny 2006: Ralph Penny. Gramática histórica del español. Barcelona: Ariel.

Pérez Saldanya 1998: Manuel Pérez Saldanya. Del llatí al català: morfosintaxi verbal històrica. València: Universitat de València.

Perlmutter 1978: David Perlmutter. «Impersonal passives and the unaccusative hypothesis». Papers from the Annual Meeting of the Berkeley Linguistic Society, 4: 157-189. Web. 10/11/2019.

Perlmutter 1989: David Perlmutter. «Multiattachment and the unaccusative hypothesis: the perfect auxiliary in Italian». Probus, 1: 63-119.

Pountain 1985: Christopher J. Pountain. «Copulas, Verbs of Possession and Auxiliaries in Old Spanish: The Evidence for Structurally Interdependent Changes». Bulletin of Hispanic Studies, 62/4: 337-355.

Ramos 2005: Joan-Rafael Ramos. «Els auxiliars dels temps compostos en català medieval». Caplletra, 38: 179-209.

Rodríguez Molina 2006: Javier Rodríguez Molina. «Ser + participio en español antiguo: perífrasis resultativa, no tiempo compuesto». José Luis Girón Alconchel \& José Jesús de Bustos Tovar (coord.), Actas del VI Congreso Internacional de Historia de la Lengua española, Madrid, 29 de septiembre-3 octubre 2003, vol. 2, Madrid: Arco/ Libros, 1059-1072.

Romani 2006: Patrizia Romani. «Tiempos de formación romance I. Los tiempos compuestos». Concepción Company Company (ed.), Sintaxis histórica de la lengua española. Primera parte: La frase verbal, México D. F.: Universidad Nacional Autónoma de México / Fondo de Cultura Económica, 243-346.

Romani 2008: Patrizia Romani. «La ambigüedad de haber+participio y ser+participio en el castellano medieval». Concepción Company Company \& José G. Moreno de Alba (eds.), Actas del VII Congreso Internacional de Historia de la Lengua Española, vol. I, Madrid: Arco / Libros, 1073-1085. 
Rosemeyer 2012: Malte Rosemeyer. «How to measure replacement: auxiliary selection in Old Spanish bibles». Folia Linguistica Historica, 33/1: 135-174.

Rosemeyer 2014: Malte Rosemeyer. Auxiliary selection in Spanish. Gradience, gradualness, and conservation. Amsterdam / Philadelphia: John Benjamins.

Sorace 2000: Antonella Sorace. «Gradients in auxiliary selection with intransitive verbs». Language, 76: 859-890. Web. 10/11/2019.

Stolova 2006: Nataliya Stolova. «Split Intransitivity in Old Spanish: Irrealis and negation factors». Revue Roumain de Linguistique, 51/2: 301320. Web. 10/11/2019.

Suárez 2016: Mercedes Suárez. «Las propiedades del sujeto y la inacusatividad de los verbos de movimiento en castellano medieval». Itinerarios: revista de estudios lingüisticos, literarios, históricos y antropológicos, 23: 155-174. Web. 10/11/2019.

Vincent: 1982: Nigel B. Vincent. «The development of the auxiliaries HABERE and ESSE in Romance». Nigel Vincent y Martin Harris (eds.), Studies in the Romance verb, London: Croom Helm, 71-96.

Yllera: 1980: Alicia Yllera. Sintaxis histórica del verbo español: las perífrasis verbales. Zaragoza: Pórtico.

\section{AUXILIARY SELECTION IN THE SEVILLIAN VERSION OF THE HISTORIA DEL NOBLE VESPASIANO}

\section{Summary}

The aim of this paper is to examine the alternation of different auxiliary verbs - that correspond to HABERE and ESSE in Latin - in constructions formed by the past participle of intransitive verbs, which, like in other languages that conserve it still today, existed also in Old Spanish. Both the conditions of this variation and the chronology of its disappearance have been widely examined but the data and the conclusions offered by the specific bibliography are sometimes contradictory. In this paper, we are going to analyse some data extracted from the Sevillian version of the Historia del noble Vespasiano (1499), a narrative text, in order to classify intransitive verbs of Old Spanish from the point of view of the auxiliary selection, paying special attention to ones that show alternation of auxiliaries accepting both of them.

Keywords: Auxiliary Selection, Compound Verbal Tenses, Old Spanish. 